\title{
Convergência dos estudos CTS e da Paisagem para entender a Relação
} Sociedade-Natureza

Convergence of STS Studies and Landscape Science to understand the Relation SocietyNature

Convergencia de los estudios CTS y del Paisaje para entender la Relación SociedadNaturaleza

Emerson Luís Pawoski da Silva Mestrando em Ciência, Tecnologia e Sociedade, IFPR-Paranaguá, Brasil. emprovoski@gmail.com

Emerson Luis Tonetti Professor Doutor, IFPR-Paranaguá, Brasil. emerson.tonetti@ifpr.edu.br 


\begin{abstract}
RESUMO
A sociedade e natureza não se encontram separadas. A desconsideração dos limites naturais em atividades humanas tem causado crises socioambientais. Compreendendo que natureza e sociedade criam o ambiente, estudos que dialoguem com esses aspectos devem ser integralizados, como o estudo da paisagem, das ciências ambientais, e a Teoria Ator-Rede, do escopo da área interdisciplinar da Ciência, Tecnologia e Sociedade (CTS), fazem. O presente estudo tem por objetivo comparar os métodos e aplicações do estudo da paisagem e da Teoria Ator-Rede para demonstrar como a CTS pode estudar interações socioambientais. A Teoria Ator-Rede é capaz de avaliar a relação entre sociedade e natureza, porque uma rede é criada quando atores, humanos e não-humanos, atuam uns sobre os outros. Os atores humanos representam a sociedade, enquanto os atores não-humanos a natureza, tecnologia, artefatos e outros. $\mathrm{O}$ estudo da paisagem compreende que um local pode ser avaliado pelo potencial ecológico ou aspectos físicos e químicos; exploração biológica ou composição e influências dos seres vivos; e ação antrópica ou transformação dos elementos naturais por sociais ou tecnológicos. Esses aspectos do estudo da paisagem estão compreendidos no Sistema Geossistema-Território-Paisagem, onde geossistema compreende principalmente os dois primeiros, território todos os três e paisagem, apenas o último. O GTP do estudo da paisagem e Teoria Ator-Rede são similares pois os diversos componentes do ambiente podem ser considerados atores, enquanto o ambiente pode ser percebido como uma rede. São precisos mais estudos para abrangência de outros tópicos da CTS quanto a interações socioambientais.
\end{abstract}

PALAVRAS-CHAVE: Teoria Ator-Rede. Ciências ambientais. Epistemologia.

\title{
ABSTRACT
}

The society and nature are not separated. The disregard of nature limits in human activities has caused socioenvironmental crisis. Sciences that study that interaction should be integrals because nature and society creates the environment sciences. The Landscape Sciences from environmental sciences and Actor-Network Theory from interdisciplinary Science, Technology and Society (STS) are able to that analysis. This study aim is compare methods and uses of Actor-Network Theory and Landscape Science to demonstrate STS application for socio-environmental interactions. The Actor-Network Theory is capable of evaluate society and nature relation since a network is conceived when human or nonhumans actors act over others. Human actor represents society while nonhuman actors are nature, technology, artifacts and others. The Landscape Sciences comprehends that a region can be evaluated by ecological potential or chemical and physical aspects; biological exploitation or biodiversity and influences of living beings; and anthropic action or transformation of nature elements to technological and social elements. Those aspects of Landscape Sciences compound the Geosystem-Territory-Paysage System (GTP). Geosystem comprehends mainly the first and second, territory is all three and paysage only the third. GTP from Landscape Sciences and Actor-Network Theory are similar since environmental components can be considered as actors and environment as a network. More studies are necessary to understand the competence of other topics of STS to socio-environmental interactions.

KEY-WORDS: Actor-Network Theory. Environmental sciences. Epistemology.

\section{RESUMEN}

La sociedad y la naturaleza no se hallan separadas. La desconsideración de los límites naturales en las actividades humanas ha causado crisis socioambientales. En cuanto a la naturaleza y la sociedad crean el ambiente, los estudios que dialoguen con estos aspectos deben ser integrados, como el estudio del paisaje, de las ciencias ambientales, y la Teoría Actor-Red, del alcance del área interdisciplinaria de la Ciencia, Tecnología y Sociedad (CTS), lo hacen. El presente estudio tiene por objetivo comparar los métodos y aplicaciones del estudio del paisaje y de la Teoría ActorRed para demostrar cómo la CTS puede estudiar interacciones socioambientales. La Teoría Actor-Red es capaz de evaluar la relación entre sociedad y naturaleza, porque una red es creada cuando actores, humanos y no humanos, actúan unos sobre otros. Los actores humanos representan a la sociedad, mientras que los actores no humanos la naturaleza, la tecnología, los artefactos y otros. El estudio del paisaje comprende que un sitio puede ser evaluado por el potencial ecológico o aspectos físicos y químicos; explotación biológica o composición e influencias de los seres vivos; y acción antrópica o transformación de los elementos naturales por sociales o tecnológicos. Estos aspectos del estudio del paisaje están comprendidos en el Sistema Geosistema-Territorio-Paisaje, donde el geosistema comprende principalmente los dos primeros, territorio los tres y el paisaje, sólo el último. EI GTP del estudio del paisaje y la Teoría 
Actores-Red son similares pues los diversos componentes del ambiente pueden ser considerados actores, mientras que el ambiente puede ser percibido como una red. Se necesitan más estudios para abarcar otros temas de la CTS en cuanto a las interacciones socioambientales.

PALABRAS CLAVE: Teoría del actor-red. Ciencias Ambientales. Epistemología. 


\section{INTRODUÇÃO}

A natureza está além das florestas e entraves econômicos, pois rios permitem a pesca, o transporte de mercadorias e pessoas, e insetos atuam na polinização de plantas alimentícias, sendo preciso mantê-la para o bem-estar humano (RICKLEFS, 2010). A sociedade não deve atuar unicamente como um extrator de recursos naturais, enquanto a natureza não pode ser conceituada apenas como matéria-prima em atividades que provêm o bem-estar (GARCIA; VISO, 2008; ACOSTA, 2016). É necessário transpassar essa dicotomia entre os elementos da sociedade e da natureza no ambiente, uma vez que esse conceito prejudica todo o sistema de interação entre esses (SERRES, 1990). A controversa e tecnológica revolução verde, através, de fertilizantes, maquinarias e biocidas permitiu crescimentos exponenciais na produção agrícola mundial, contudo, removeu nutrientes básicos à planta cultivada e os agentes tóxicos apresentaram danos a saúde e ao ambiente (RICKLEFS, 2010). As ciências, e áreas de conhecimento, que estudam o ambiente, devem possuir uma visão sistêmica capaz de localizar as associações entre seus componentes, evitando a noção de que não há efeitos sequenciais (HARVEY, 2007).

A relação entre sociedade e natureza é academicamente bastante dificultada pelas diferenças entre ciências naturais e humanas. No entanto, sendo preciso uma epistemologia integralizada, devido à interação dos fatores sociais e naturais no ambiente (LATOUR, 2013). O ambiente é concebido como o conjunto de componentes físicos, químicos e biológicos, isto é a natureza, de um local, que pode ser habitado, influenciado, ao mesmo tempo em que influencia a sociedade (BERTRAND; BERTRAND, 2014; PASSOS, 2017). O estudo da paisagem, um método da geografia, ganhou destaque na década de 1970 (BERTRAND, 2004) para avaliar o ambiente através das relações entre sociedade e natureza (WULF, 2016).

Para o naturalista alemão Alexander von Humboldt, um dos pioneiros em estudos ambientais, influenciador do estudo da paisagem e sob a indução do texto "Critica da Razão Pura" de Immanuel Kant, era preciso considerar as restrições do cientista para avaliar o conhecimento produzido (WULF, 2016) e essa necessidade também está presente em uma área de estudos interdisciplinares chamada Ciência, Tecnologia e Sociedade (PALACIOS et al, 2003). A Ciência, Tecnologia e Sociedade é uma que compreende a ciência como uma criação social, a tecnologia como uma extensão mediada pela ciência do ser humano e a sociedade contemporânea como resultado da ciência e tecnologia. Desse modo, entende-se que as limitações dos cientistas e dos momentos históricos, bem como a sociedade e natureza, influenciam e são influenciadas pela ciência e tecnologia (PALACIOS et al, 2003; SANTOS, 2003; BACHELARD, 2005). A Teoria AtorRede é um conceito da CTS, bastante promissor na busca de relacionar os elementos naturais e sociais considerando-os atores em uma rede de interações (BECK et al, 2017).A hipótese de que a CTS permite uma epistemologia integralizada sobre o ambiente e oferece um diálogo entre as ciências naturais e humanas, similar a algumas linhas das ciências ambientais, parece intuitiva. 


\section{METODOLOGIA}

A relação entre sociedade e natureza, através da CTS e estudos ambientais, foi verificada na literatura. O texto destaca e compara pontos relevantes da Teoria Ator-Rede (do escopo da CTS) e do estudo da Paisagem (do escopo das Ciências Naturais) para demonstrar convergências metodológicas. O presente estudo tem caráter bibliográfico e foi fundamentado na busca de palavras-chave, como paisagem; geossistema; teoria ator-rede e outras similares em indexadores da Capes e Scielo. Para isso, a presente pesquisa foi organizada em apresentação da Teoria Ator-Rede do escopo CTS e a possibilidade da mesma para relacionar sociedade e natureza; demonstração do estudo da paisagem, aproximando a natureza dos aspectos humanos e da tecnologia; e similaridades entre o estudo da paisagem e a Teoria Ator-Rede para destacar como a CTS pode realizar estudos ambientais integralizados.

\section{RESULTADOS E DISCUSSÃO}

\subsection{A relação sociedade e natureza mediada pela Teoria Ator-Rede}

O compartilhar de recursos hídricos poluídos e produção de transgênicos são exemplos de agentes e eventos híbridos entre a sociedade e natureza, sendo capazes de atuar sobre seus geradores. A inclusão da natureza sobre discussões políticas é vital devido à constância e implicações dos componentes naturais sobre a sociedade. Para compreender as crises socioambientais na produção de alimentos, energia, poluição, uso e ocupação da terra é preciso haver uma democracia onde os atores humanos e não-humanos, como os elementos naturais água, fauna e flora sejam expostos e o conhecimento científico é uma das melhores formas dessa representação, em decorrência de sua rigorosidade de método e analise. Entre as representações mais comuns estão os estudos do clima e da biodiversidade que indicam que $o$ progresso cientifico e tecnológico não afeta apenas humanos e sim todo o ambiente (LATOUR, 2013).

Nos estudos interdisciplinares CTS, por exemplo, evidencia-se a ideia da coprodução de objetos e fenômenos, onde ciência, tecnologia, política e natureza interferem-se e co-evoluem e, assim, os dados ambientais são demonstrativos dos efeitos da ciência e tecnologia na sociedade. 0 livro "The Handbook of Science e Technology Studies", apresenta a Teoria Ator-Rede como uma ferramenta para entender essas relações (BECK et al, 2017), e essa demonstra que não se deve condenar ou idolatrar, mas, discutir a tecnologia, considerando as peculiaridades de seus componentes, os artefatos, como evidenciado pelos principais teóricos dessa teoria, Bruno Latour, John Law e Michel Callon (DAl; HAO, 2018).

O conceito de artefato é bastante amplo, porém, seu significado pode ser um termo em comum entre diferentes ciências e áreas do conhecimento. A palavra artefato tem origem na expressão "artus factum" e representa produtos fabricados, construídos ou modelos por uma técnica e que mediam a interação entre sujeito, com seus objetivos, e um determinado objeto ou fenômeno (BERTRAND; BERTRAND, 2014). Os artefatos expandem ou selecionam as ações 
humanas e seus objetivos, podendo auxiliar a tomar decisões que afetem o cotidiano. Pode-se afirmar que os artefatos se relacionam com o ser humano e lhe proporcionam um modo de existência sócio-técnico (SIMONDON, 2007) onde a distribuição espacial desses elementos é muito importante. Se artefatos forem considerados objetos técnicos, podem ser classificados em elementos técnicos que representam ferramentas e os indivíduos técnicos são máquinas ou seres vivos que são capazes de agir sem a influência humana (QUEIROZ-MELO; MORAES, 2016). Pela perspectiva da capacidade de indução, dominação e privilégio oferecida por artefatos a sociedade, pode haver compensações em suas funções, onde podem aprimorar ou dificultar determinado modo de existência (LATOUR, 1994). Os processos sociais que produzam a ineficácia de técnicas e artefatos são tão importantes quanto os que produzem sua eficácia e a consideração com a mesma relevância, chamada simetria, desses permite um estudo mais consciente nos estudos de ciência e tecnologia (BLOOR, 2003). O princípio da simetria, modificado do conceito de David Bloor por Bruno Latour, permite comunicar, além da ciência e tecnologia com a sociedade, os artefatos, locais, animais não-humanos e demais seres vivos, aumentando ou reduzindo a importância desses. Essas diferentes personagens do cotidiano podem ser considerados atores, ou seja, entidades atuantes, que interferem uns aos outros em uma rede de relações. Essas relações ocorrem quando há objetivos em comuns ou intercalados (LATOUR, 1994) como a necessidade humana de obtenção de frutos, que depende da polinização de abelhas, onde essas precisam de uma diversidade de recursos florais do ambiente para manter suas colônias.

Os atores não-humanos são bastantes uteis nas discussões políticas e sociais sobre essas associações sócio-técnicas por que não apresentam preconceitos, ideologias e demais aspectos semelhantes (LATOUR, 2013). Se através do conhecimento científico descobre-se que é preciso considerar e respeitar as particularidades naturais, então a natureza deve ser percebida como ator nessas relações. Esse princípio também está presente nas ciências ambientais, cujos estudos compreendem os conflitos socioambientais e a gestão dos recursos naturais (LATOUR, 1994), na busca por maior sustentabilidade.

Para entender esses sistemas de interações que ocorrem em determinado local, nas ciências ambientais, é utilizado do estudo da paisagem (NEVES et al, 2014), que apresenta similaridades a Teoria Ator-Rede ao realizar uma simetria dos componentes que constituem esse local.

\subsection{0 estudo da paisagem}

A Teoria Geral dos Sistemas (TGS) proposta por Ludwig Von Bertalanffy, em 1930 busca compreender uma totalidade pelo estudo e relações de suas partes, compostas de uma estrutura especifica e função relativa a outras partes. A ecologia se beneficiou bastante da TGS para estudar os ambientes e seus componentes. O ecossistema é um ambiente e condição onde fatores bióticos, como comunidades, e abióticos, como solo e clima, se relacionam e há uma ciclagem dos nutrientes entre esses fatores (NUCCl, 2007).

Devido à necessidade de especialização de disciplinas, a ecologia passou, com maior veemência a partir de 1974, a estudar a relação entre os seres vivos e desses com o ambiente, com certo 
grau de isolamento do ser humano. A pesquisa sobre os sistemas de relação, da biologia, recebeu acréscimo da geografia, originando a ecologia da paisagem, onde se inclui o ser humano como parte do ecossistema ( $\mathrm{NUCCl}, 2007)$, aproximando do conceito de geossistema no estudo da paisagem (Quadro 1) (NEVES et al, 2014).

Quadro 1: Comparação entre abordagens pelas compreensões ecossistêmicas e geossistêmicas.

\begin{tabular}{|c|c|c|}
\hline Tipo de estudo & Ecossistema & Geossistema \\
\hline Fauna e Flora & $\begin{array}{c}\text { Ecologia de plantas e } \\
\text { animais }\end{array}$ & $\begin{array}{c}\text { Relação de plantas e } \\
\text { animais com a degradação } \\
\text { ambiental e atividades } \\
\text { socioeconômicas }\end{array}$ \\
\hline Área & Irrelevante & $\begin{array}{c}\text { Descrita em escalas } \\
\text { consoante unidades da } \\
\text { paisagem }\end{array}$ \\
\hline Ser humano & $\begin{array}{c}\text { Comparação entre } \\
\text { ambientes naturais e } \\
\text { antropizados }\end{array}$ & $\begin{array}{c}\text { Compreensão da dinâmica } \\
\text { dos componentes naturais } \\
\text { e antrópicos no ambiente }\end{array}$ \\
\hline
\end{tabular}

Fonte: NEVES et al, 2014.

Os sistemas de interação podem ser mais fechados pelas suas propriedades e distâncias ou mais abertos, em que as dinâmicas desses provem dos fatores naturais e fatores antrópicos territorialidade e atividade econômica (SANTOS, 2003; GUERRA; SOUZA; LUSTOSA, 2012). 0 geossistema é composto por três partes: o potencial ecológico, isto é, o solo, água, ar e clima; exploração biológica ou atuação e existência da biota; ação antrópica, ou seja, a interferência no ambiente pelo ser humano. O geossistema, proposto por Georges Bertrand na década de 1970, se propõe a estudar o ambiente através de categorias de área em dezenas, centenas, milhares e demais metros quadrados (LOPES; SILVA; GOULART, 2014).

Para facilitar esses estudos, Monteiro (2001) propõe a substituição dos níveis taxonômicos do espaço físico pelo uso das próprias áreas estudadas, sendo assim denominadas unidades da paisagem em variadas escalas. A paisagem é proposta como uma entidade espaço-temporal em que recursos do ambiente são utilizados e depositados por agentes animais, vegetais, microbiológicos e por seres humanos (BERTRAND, 2004). A humanidade é diferenciada dos demais seres vivos pelo tipo e intensidade de suas interações. $O$ estudo é baseado em modelos que analisam solo, vegetação, clima, entre outros que caracterizam uma área chamada unidade da paisagem (MONTEIRO, 2001).

Esse método de análise compreende o ser humano como igual a outros animais, plantas e clima na construção do ambiente. Contudo o impacto da ação humana é muito mais intenso e tem caráter sócio histórico. Em uma tentativa de contemplar esse contexto, Georges Bertrand e Claude Bertand, em 2007, propõem o Sistema GTP ou Geossistema-Território-Paisagem (GUERRA; SOUZA; LUSTOSA, 2012).

Nesse sistema um mesmo ambiente pode ser percebido como: geossistema ou expressão do funcionamento biofísico com dominância de avaliações do potencial ecológico e exploração biológica; território ou tecnologia que avalia a natureza, isto é, potencial ecológico e exploração biológica, na mesma proporção que a ação antrópica; paisagem ou representação sensível, onde 
há maior importância da ação antrópica (NEVES, 2017). O geossistema é visto como a fonte de recursos ou espaços, o território como o uso do geossistema como recurso, isto é, o que é extraído ou depositado e como se relaciona com a economia e sociedade, e a paisagem é a identidade cultural do espaço oriunda da percepção humana (GUERRA; SOUZA; LUSTOSA, 2012), podendo ser dados diferentes enfoques que estão ligados as necessidades e capacidades dos cientistas ambientais (NEVES, 2017).

O estudo da paisagem no Brasil tem sido bastante útil na compreensão, intervenção cientifica e resolução de questões socioambientais (MONTEIRO, 2001; AB'SABER, 2003). No ambiente, os componentes naturais influenciam a sociedade, que por sua vez se organiza e remodelam esses consoantes seus objetivos (NEVES et al, 2014; ADLER; TANNER, 2015). Dada as possíveis semelhanças teóricas entre a Teoria Ator-Rede e o estudo da paisagem das ciências ambientais, pode-se demonstrar que a interdisciplinar CTS é capaz de avaliar a relação sociedade e natureza. Nesse sentido a apresentação das comparações entre esses métodos facilita essa concepção para diferentes categorias de cientistas.

\subsection{O estudo da paisagem e a Teoria Ator-Rede}

A sociedade utiliza da tecnologia para domínio da natureza, a compreensão de deslocamento desses três aspectos causa a insustentabilidade de modos de vida (GARCIA; VISO, 2008). Intensos e inconsequentes usos da terra e extração dos recursos naturais alteram a estabilidade do ambiente e prejudicam esse sistema de relação entre ser humano e natureza (BERTRAND; BERTRAND, 2014).

Contrariando a premissa que apenas a sociedade cria o ambiente (HARVEY, 2007), a ideia de uma construção comum entre atores humanos e não-humanos é antiga, mas, ainda é necessário o desenvolvimento e melhoramento de métodos que permitam fazer essa analise (VITTE, 2012). A dificuldade de avaliar o ambiente, bem como torná-lo sensível (BERTRAND; BERTRAND, 2014) e os conflitos socioambientais, promoveram a criação de diferentes epistemologias (SANTOS, 2003). A maioria das pesquisas socioambientais foram realizadas no hemisfério norte, onde se distribuem estudos com gestão, qualidade, legislação e planejamento ambiental (KABISCH; QURESHI; HAASE, 2015). A concepção ambiental varia de acordo com a cultura e objetivo dos observadores. Cientistas ambientais podem relacionar várias regiões em suas abordagens, diferentemente de outras pessoas que não estejam ligadas a essa área do conhecimento. (BARBOSA; NASCIMENTO-JÚNIOR, 2009).

O Sistema GTP pode, por exemplo, estudar riscos ambientais em relação a tecnologia (Figura 1) tratando os geossistemas como riscos biofísicos, território como riscos sócio-técnicos e a paisagem como a percepção da população desses (NEVES; MACHADO; CAMARGO, 2017). 
Figura 1 - Relação entre geossistema, território e paisagem na percepção de riscos ambientais.

\begin{tabular}{|c|c|c|c|}
\hline & & & Percepção (Paisagem) \\
\hline Conceitos & Sub-conceitos & Variaveis & \\
\hline \multirow{7}{*}{$\begin{array}{c}\text { Perigo } \\
\text { (Geossistema) }\end{array}$} & \multirow{4}{*}{ Riscos físicos } & Erosão & \\
\hline & & Escorregamento & \\
\hline & & Assoreamento & \\
\hline & & Inundação-Cheia & \\
\hline & \multirow{3}{*}{$\begin{array}{l}\text { Riscos } \\
\text { biológicos }\end{array}$} & Alterações na fauna & \\
\hline & & Alterrações na flora & \\
\hline & & Ploriferação de vetores & \\
\hline \multirow{11}{*}{$\begin{array}{c}\text { Vulnerabilidade } \\
\text { (Território) }\end{array}$} & \multirow{8}{*}{ Riscos sociais } & Lixo & \\
\hline & & Infraestrutura das casas & \\
\hline & & Infraestrutura urbana & \\
\hline & & Danos a bens edificados & \\
\hline & & Condições sanitárias/ esgoto & \\
\hline & & Ruído/ barulho & \\
\hline & & Ocupação irregular & \\
\hline & & Doenças & \\
\hline & \multirow{3}{*}{$\begin{array}{c}\text { Riscos } \\
\text { tecnológicos }\end{array}$} & Poluição do ar & \\
\hline & & Materiais inflamaveís & \\
\hline & & Poluição da água & \\
\hline
\end{tabular}

Fonte: NEVES; MACHADO; CAMARGO, 2017.

Os elementos do estudo da paisagem têm semelhantes na Teoria Ator-Rede da CTS. Aspectos naturais como clima, solo, água, território, economia e demais seres vivos podem ser compreendidos como atores não-humanos, enquanto as pessoas que vivem com esses são os atores humanos (LATOUR, 2013; BECK et al, 2017). A interdisciplinar CTS também oferece assim, através da Teoria Ator-Rede, um modo de se estudar a sustentabilidade nos espaços compartilhados pela natureza e sociedade. Nas cidades, por exemplo, o planejamento do ambiente urbano, sob essa perspectiva, descreve que os elementos naturais e sociais transformam, como atores, o ambiente (BIBRI; KROGSTIE, 2017). Enquanto a cidade, que serve como instrumento de dominação sobre a natureza, pode ser concebida como uma tecnologia (PALACIOS et al, 2003).

A interação que ocorre entre esses componentes determina uma rede (LATOUR, 2012) e um ambiente (MONTEIRO, 2001). Esse sistema ambiental não pode ser considerado fechado, uma vez que, os componentes e associações entre esses mudam ao longo do tempo (SANTOS, 2003), como ocorre às redes de atores (CALLON, 1995; LATOUR, 1994).

Se o ambiente é um produto da sociedade e natureza, os estudos de ambientes devem compreender essas interações. Ciências sociais e naturais apresentam diferentes metodologias para esse fim, contudo, como exposto, a Teoria Ator-Rede e o estudo da paisagem são capazes de realizar esse diálogo (Figura 2). 


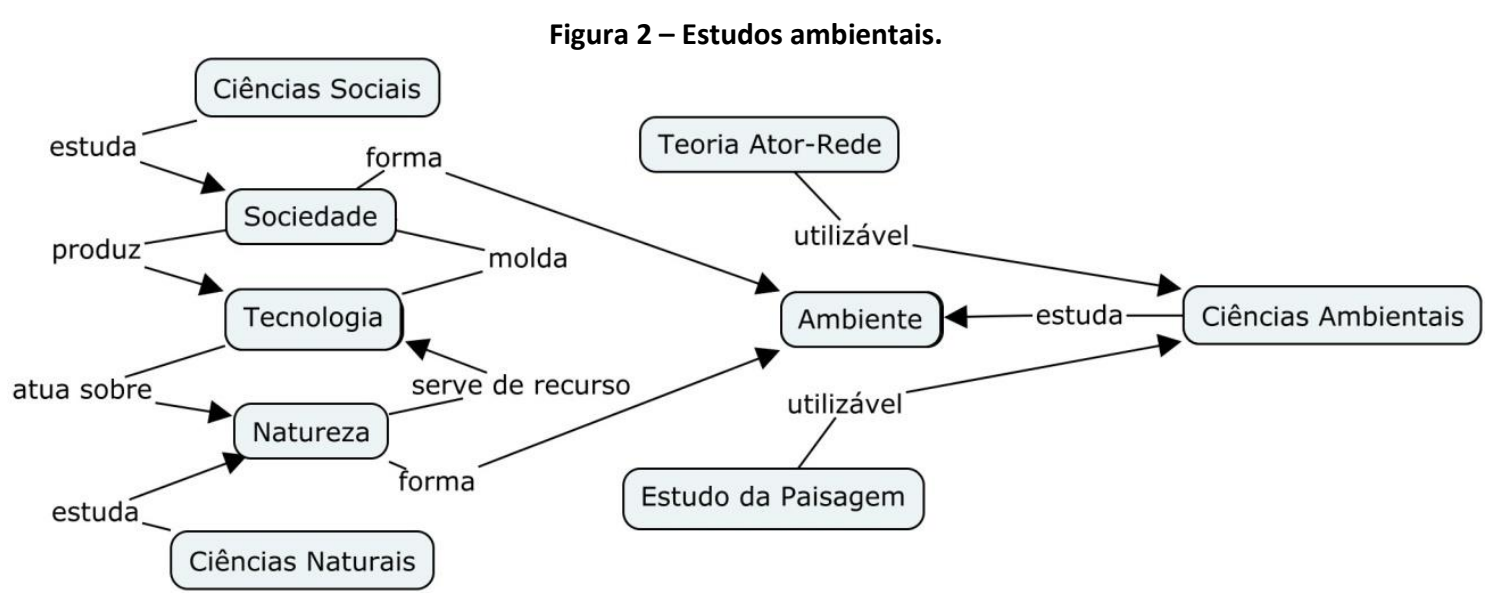

Fonte: Os autores

A interdisciplinar CTS apresenta uma epistemologia integralizada entre sociedade e natureza, semelhante ao estudo da paisagem, sendo capaz de promover a sustentabilidade ao relacionar as necessidades de cada componente ambiental (GARCIA; VISO, 2008; ERAGHI; MESCHI; GHOLAMPOUR, 2015; PASSOS, 2017).

\section{CONSIDERAÇÕES FINAIS}

Os recursos ambientais são estudados e utilizados conforme determinados conhecimentos prévios e objetivos de pesquisadores ( $\mathrm{NUCCl}, 2007)$, havendo muitas epistemologias para diferentes grupos sociais (BACHELARD, 2005). Para Xiangyu e Youxi (2013), a especialização e modo de uso das ciências e tecnologias provocam a dicotomia entre sociedade e natureza, sendo necessária uma fundamentação integralizada e ecológica dessas para a sustentabilidade. No presente estudo, apresentou-se a Teoria Ator-Rede, que faz parte do escopo da área interdisciplinar da Ciência, Tecnologia e Sociedade (BECK et al, 2017), e estudo da paisagem, que está compreendida entre a ecologia e geografia ( $\mathrm{NUCCl}, 2007)$, como métodos capazes de estudar elementos humanos e naturais. Essas abordagens apresentam similaridades em seus instrumentos e limitações, como é o caso da perspectiva do cientista (PALACIOS et al, 2003; WULF, 2016).

As indicações da literatura demonstram o aplicável e fácil uso da Teoria Ator-Rede, no entanto, outras linhas de pesquisa da CTS podem relacionar sociedade e natureza em um sistema, sendo precisos outros estudos comparativos para essa verificação.

\section{REFERÊNCIAS BIBLIOGRÁFICAS}

$A B$ 'SABER, A. N. Os domínios de natureza no Brasil: potencialidades paisagísticas. São Paulo: Ateliê Editorial, 2003.

ACOSTA, A. O bem viver: uma oportunidade para imaginar outros mundos. Autonomia Literária, 2016. 
ADLER, F. R.; TANNER, C. J. Ecossistemas urbanos: princípios ecológicos para o ambiente construído. São Paulo: Oficina de Textos, 2015.

BACHELARD, G. A formação do espírito científico: contribuição para uma psicanálise do conhecimento. Rio de Janeiro: Contraponto, 2005.

BARBOSA, V.L.; NASCIMENTO-JÚNIOR, A.F. Paisagem, Ecologia Urbana e Planejamento Ambiental. Geografia, v.18, n.2, p.21-36, 2009.

BECK, S.; FORSYTH, T.; KOHLER, P.M.; LAHSEN, M.; MAHONY, M. The Making of Global Environmental Science and Politics. In: FELT, U.; FOUCHÉ, R.; MILLER, C.A.; SMITH-DOERR, L. (eds). The handbook of science and technology studies. 4 ed. Cambridge MA: MIT Press, 2017, p.1059-1086.

BERTRAND, G. Paisagem e Geografia Física Global- Esboço metodológico. Revista Ra'e Ga, v.8, p.141152, 2004.

BERTRAND, C.; BERTRAND, G. La Nature-Artefact: entre Anthropisation et Artialisation, l'expérience du Système GTP (Géosystème-Territoire-Paysage). Information géographique, v.78, n.3, p.10-25, 2014.

BIBRI, S. E.; KROGSTIE, J. On the Social Shaping Dimensions of Smart Sustainable Cities: A Study in Science, Technology, and Society. Sustainable Cities and Society, v.29, n.1, p.219-248, 2017.

BLOOR, D. Conocimiento e Imaginario Social. Barcelona: Gedisa Editorial, 2003.

CALLON, M. Technological conception and adoption network: lessons for the CTA practitioner. In: RIP, A.; MISA, T.; SCHOT, J. (eds). Managing technology in society: The approach of Constructice Technology Assessment. Londres: Pinter, 1995, p.307-330.

DAI, Y.; HAO, S. Transcending the opposition between techno-utopianism and techno-dystopianism. Technology in Society, v.53, n.1, p.9-13, 2018.

ERAGHI, S.G.; MESCHI, M.; GHOLAMPOUR, S. Studying the Relationship Between Urban Green Corridors and Sustainable Urban Landscape. International Journal of Science, Technology and Society, v.3, n.2-1, p.36-40, 2015.

FINO, F.F. Diseño metodológico para la definición de determinantes ambientales regionales basadas en el sistema GTP en la jurisdicción de CORPOCHIVOR. Perspectiva Geográfica, v.16, n1, p.55-81, 2011.

GARCIA, M.G.; VISO, A.M. Ciencia, tecnología y sostenibilidad. Revista Iberoamericana de Ciencia, Tecnología y Sociedad - CTS, v.4, n.11, p. 47-51, 2008.

GUERRA, M.D.F.; SOUZA, M.J.N.; LUSTOSA, J.P.G. Revisitando a Teoria Geossistêmica De Bertrand no Século XXI: aportes para O GTP (?). Geografia em Questão, v.5, n.2, p.28-42, 2012.

HARVEY, D. Condição pós-moderna: Uma Pesquisa sobre as Origens do Mundo Cultural. 16 ed. São Paulo: Loyola, 2007.

KABISCH, N.; QURESHI,S.; HAASE,D. Human-environment interactions in urban green spaces - A systematic review of contemporary issues and prospects for future research. Environmental Impact Assessment Review, v.50, n.1, p.25-34, 2015.

LATOUR, B. On technical mediation - philosophy, sociology, genealogy. Common Knowledge, v.3, n.2, p.29-64, 1994. 
LATOUR, B. Reagregando o Social: uma introdução à teoria do Ator-Rede. Salvador: Edufba, 2012.

LATOUR, B. Jamais fomos modernos. 3 ed. Rio de Janeiro: Editora 34, 2013.

LOPES, L.G.N.; SILVA, A.G.; GOULART, A.C. Novos caminhos na análise integrada da paisagem: abordagem geossistêmica. Natureza on line, v.12, n.4, p.156-159, 2014.

MONTEIRO, C. A. F. Geossistemas: a História de uma Procura. 2 ed. São Paulo: Contexto, 2001.

NEVES, C.E. A geografia desconhecida de Georges Bertrand: contribuições à discussão e aplicação do “Geossistema Complexo" no Brasil. Revista Estudos Geográficos, n.15, p.139-166, 2017.

NEVES, C.E.; MACHADO, G.; CAMARGO, K.C. Subsídio do sistema GTP (Geossistema- TerritórioPaisagem) na percepção de riscos ambientais: esboço metodológico. Geografia, v.26, n.1, p.76-91, 2017.

NEVES, C.E.; MACHADO, G.; HIRATA, C.A.; STIPP, N.A.F. A importância dos geossistêmas na pesquisa geográfica: uma análise a partir da correlação com o ecossistema. Sociedade e Natureza, v.26, n.2, p.271-285, 2014.

NUCCl, J.R. Origem e desenvolvimento da ecologia e da ecologia da paisagem. Revista Eletrônica Geografar, v.2, n.1, p.77-99, 2007.

PALACIOS, E.M.G.; LINSINGEN, I.; GALBARTE, J.C.G.; CEREZO, J.A.L.; LUJÁN, J.L.; PEREIRA, L.T.V.; GORDILLO, M.M.; OSORIO, C.; VALDÉS, C.; BAZZO, W.A. (eds.). Introdução aos Estudos CTS: Ciência, tecnologia e sociedade. Cadernos de Ibero-América, Editora OEI, 2003.

PASSOS, M.M. A paisagem, uma ferramenta de análise de territórios emergentes na interface entre natureza e sociedade: o vale do Guaporé - Jauru/MT-Brasil. Cadernos de Geografia, n.36, p. 27-45, 2017.

QUEIROZ-MELO, M. F. A.; MORAES, M. O. A técnica como modo de existência: um diálogo entre as ideias de Latour e Simondon. Memorandum, v.31, n.1, p.276-297, 2016.

RICKLEFS, R.E. A Economia da Natureza. 6 ed. Rio de Janeiro: Guanabara Koogan, 2010.

SANTOS, B.S. Introdução a uma ciência pós-moderna. Rio de Janeiro: Graal, 2003.

SERRES, M. O contrato natural. Lisboa: Instituto PIAGET, 1990.

SIMONDON, G. El modo de existencia de los objetcos técnicos. Buenos Aires: Prometeo Libros, 2007.

VITTE, A.C. Da Transvalorização dos conceitos a uma nova proposta de geossistêmica para à abordagem de uma natureza híbrida. Revista Geonorte, v.4, n.4, p.1 - 21, 2012.

WULF, A. A invenção da natureza: a vida e as descobertas de Alexander von Humboldt. São Paulo: Planeta, 2016.

XIANGYU, Y.; YOUXI, Y. On the environmental and technology aesthetics and the innovation and development of modern science and technology. International Journal of Science, Technology and Society, v.1, n.2, p.42-47, 2013. 